\title{
The Type-Agent Core for Exchange Economies with Asymmetric Information
}

\author{
Geoffroy de Clippel ${ }^{1}$ \\ Chargé de Recherches FNRS. Department of Economics, Box B, Brown \\ University, Providence RI 02912, USA.
}

\begin{abstract}
The type-agent core is a new solution concept for exchange economies with asymmetric information. It coincides with the set of equilibrium outcomes of a simple competitive screening game. Uninformed intermediaries help the agents to cooperate in an attempt to make some profit. The paper extends the work of Perez-Castrillo (1994) to exchange economies with non-transferable utility and asymmetric information. The type-agent core is a subset of Wilson (1978)'s coarse core. It is never empty, even though it may be a strict subset of Wilson's fine core. In addition, it converges towards the set of constrained market equilibria as the economy is replicated.
\end{abstract}

\section{Introduction}

The objective of this paper is to study the allocation of scarce resources between agents that are asymmetrically informed about the fundamentals of the economy. I assume for simplicity that the true state of the economy is verifiable when the contracts are implemented. Incentive and measurability constraints are therefore irrelevant.

The main reference for core concepts is Wilson (1978). An agreement specifies a way to split the endowment of the economy among the agents in each state. Such a function is called a feasible allocation rule. Wilson discusses various notions of objection against given feasible allocation rules. They differ by the amount of communication that is permitted between the agents. Two

Email address: declippel@brown.edu (Geoffroy de Clippel ).

1 Special thanks go to Professors Enrico Minelli, Herakles Polemarchakis, Roberto Serrano and Rajiv Vohra for insightful discussions; the usual disclaimer applies. 
polar notions emerged: coarse objections are based on events that are common knowledge among the members of the coalition; fine objections are based on events that can be discerned by pooling the information of the members of the coalition. Every coarse objection is a fine objection. Hence the fine core is a subset of the coarse core.

The agents are cooperating on their own in Wilson's theory. Objections emerge from coalitions. I study an alternative approach where uninformed intermediaries help the agents to coordinate in an attempt to make some profit. They compete a la Bertrand. The intermediaries correctly anticipate the set of agents that are going to buy the contracts (net trade vectors) they offer. Even without communication, this set may vary with the future state of the economy as each agent's decision is based on his own private information. This leads to an endogenous determination of the coalition that is going to form as a function of the state. In other words, I extend the competitive screening argument of Rothschild and Stiglitz (1976) to coalition formation.

The very presence of asymmetric information implies that the agents will have difficulty to perform mutually beneficial exchange and insurance without the help of outside institutions. Perez-Castrillo (1994) observes:

It is easy to find many situations in which, in order to achieve cooperation, coalitions are formed by the initiative of its own members. (...) On many other occasions, however, it is difficult for the agents to join a coalition. Information problems between agents, absence of communication mechanisms, or just the incentive problems that arise when people try to work cooperatively can make the formation of coalitions difficult. In these cases, 'exogenous' agents or institutions may be interested in achieving the emergence of these groups. Given that a coalition can generate profits, it seems that it should be possible to find a payment scheme that will make it worthwhile for the agents to join the group. When there are several exogenous agents that may be interested in creating such groups, they will compete in order to attract agents to their coalitions. Solution concepts from noncooperative game theory are then appropriate for the analysis of this type of competition.

My paper extends the results of Perez-Castrillo (1994) to exchange economies with non transferable utility and asymmetric information.

The set of subgame perfect equilibrium outcomes associated with the competitive screening game constitutes a natural notion of core. I prove that it coincides with the core (as usually defined thanks to the Arrow-Debreu contingent goods) of a fictitious exchange economy with symmetric information. The fictitious agents are defined as in the type-agent representation of Bayesian games suggested by Harsanyi (1967-68) in order to define the concept of Bayesian 
equilibrium. My new notion of core is therefore called the type-agent core.

The type-agent core is a subset of the coarse core. I propose an example where it is even a strict subset of the fine core. Although the fine core may be empty, the type-agent core is never empty. Wilson (1978, footnote 6) defines a notion of constrained market equilibrium as a technical tool to prove the non-emptiness of the coarse core. I show that, under mild conditions, the set of constrained market equilibria is a subset of the type-agent core and that the type-agent core shrinks towards the set of constrained market equilibria as the economy is replicated. Such a convergence result is rather unexpected as Serrano et al. (2001) show that neither the coarse nor the fine cores converge towards the set of constrained market equilibria when the economy is replicated. More than that, they show that the negative result is robust against many alternative definitions of both the core and the price equilibria.

\section{Framework}

The model is the same as in Wilson (1978). Let $N$ be the finite set of agents. Let $L$ be the finite set of goods. The future state of the economy is uncertain. Let $\Omega$ be the finite set of possible states. Let $\pi$ be the common prior that describes the relative probability of those states. I assume without loss of generality that $\pi(\omega)>0$ for each $\omega \in \Omega$. The agents may have some private information. The information of agent $i$ is summarized by a partition $\mathcal{P}_{i}$ of the set $\Omega$. For each $\omega \in \Omega$, let $P_{i}(\omega)$ be the atom of the partition that contains $\omega$. The interpretation goes as follows. When the future state of the economy is $\omega$, agent $i$ knows and only knows that it will be an element of $P_{i}(\omega)$. His beliefs are derived from $\pi$ by Bayesian updating. Events are subsets of $\Omega$. The probability $\pi(\omega \mid \mathcal{E})$ of any state $\omega$ given an event $\mathcal{E}$ equals 0 if $\omega \in \Omega \backslash \mathcal{E}$ and equals $\pi(\omega) / \pi(\mathcal{E})$ if $\omega \in \mathcal{E}$. The true state of the economy is common knowledge among the agents at some future date. It determines their preferences and endowments. Let $e_{i}: \Omega \rightarrow \mathbb{R}_{+}^{L}$ be the function that specifies the initial endowment of agent $i$ and let $u_{i}: \mathbb{R}_{+}^{L} \times \Omega \rightarrow \mathbb{R}$ be the function that specifies his preferences. The agents maximize their expected utilities when facing some uncertainty. The utility function of each agent is strongly increasing $\left(x^{\prime} \gtrless x\right.$ implies $u_{i}\left(x^{\prime}, \omega\right)>u_{i}(x, \omega)$ for each $\left.\omega \in \Omega\right)$, continuous and concave in each state of the economy. Decisions are taken today about the way to redistribute the endowments when the state will be common knowledge. An allocation rule is a function $a: \Omega \rightarrow \mathbb{R}_{+}^{L \times N}$. It is feasible if $\sum_{i \in N} a_{i}(\omega) \leq \sum_{i \in N} e_{i}(\omega)$ for each $\omega \in \Omega$. There are typically opportunities for insurance, even if the agents are asymmetrically informed. The expected utility of agent $i$ for some allocation rule $a$ conditional on some event $\mathcal{E}$ is $E\left(u_{i}\left(a_{i}\right) \mid \mathcal{E}\right)=\sum_{\omega \in \Omega} \pi(\omega \mid \mathcal{E}) u_{i}\left(a_{i}(\omega), \omega\right)$. The model boils down to a classical exchange economy when there is only one possible state. It coincides with the traditional model of exchange economies 
with uncertainty and symmetric information when $\mathcal{P}_{i}=\{\Omega\}$ for each $i \in N$.

\section{Definition}

Let $a$ be an allocation rule that one sees as a potential outcome. I test it against some alternative allocation rule $a^{\prime}$. Let $D\left(a, a^{\prime}, \omega\right)$ denote the set of deviators should $\omega$ be the future state of the economy. It is the set of agents that prefer (given their private information) to receive $a^{\prime}$ instead of keeping $a$ :

$$
D\left(a, a^{\prime}, \omega\right):=\left\{i \in N \mid E\left(u_{i}\left(a_{i}^{\prime}\right) \mid P_{i}(\omega)\right)>E\left(u_{i}\left(a_{i}\right) \mid P_{i}(\omega)\right)\right\}
$$

for each $\omega \in \Omega$.

The allocation rule $a^{\prime}$ is strictly feasible when proposed against $a$ if

$$
\sum_{i \in D\left(a, a^{\prime}, \omega\right)} a_{i}^{\prime}(\omega) \leq \sum_{i \in D\left(a, a^{\prime}, \omega\right)} e_{i}(\omega)
$$

for each $\omega \in \Omega$, the inequality being strict for some $\omega \in \Omega$.

An allocation rule $a$ is blocked if there exists an allocation rule $a^{\prime}$ that is strictly feasible when proposed against $a$. A dummy player with no endowment and no information can obtain a strictly positive amount of some good in some state of the economy by challenging $a$ and proposing $a^{\prime}$.

The type-agent core is the set of feasible allocation rules that are not blocked. Observe that the type-agent core coincides with the usual notion of core when there is no uncertainty (i.e. $\# \Omega=1$ ).

I will show in the next section how the type-agent core emerges from a simple competitive screening game. Before doing that, I justify the terminology. I adapt the idea of Harsanyi (1967-68), who defines a Bayesian equilibrium to be any Nash equilibrium of the type-agent representation of the original Bayesian game. I suggest the following type-agent representation of the economy described in section 2. It is a (fictitious) exchange economy with uncertainty and symmetric information. Let $\mathcal{N}$ be the set of type-agents. A type-agent is a couple $(i, \mathcal{E})$ where $i$ is an agent and $\mathcal{E}$ is an atom of his information partition. The set of goods is $L$. The set of possible states is $\Omega$. The probability of each state is determined by $\pi$. The endowment of type-agent $(i, \mathcal{E})$ in state $\omega$ equals $e_{i}(\omega)$ if $\omega \in \mathcal{E}$ and equals zero otherwise. The utility of type-agent $(i, \mathcal{E})$ for bundle $x \in \mathbb{R}_{+}^{L}$ at $\omega$ equals $u_{i}(x, \omega)$ if $\omega \in \mathcal{E}$ and equals zero otherwise. An allocation rule is a function $\mathfrak{a}: \Omega \rightarrow \mathbb{R}_{+}^{L \times \mathcal{N}}$. The associated allocation rule in the original economy is $a$ where $a_{i}(\omega)=\mathfrak{a}_{\left(i, P_{i}(\omega)\right)}(\omega)$ for each $i \in N$ and each $\omega \in \Omega$. Similarly, the allocation rule $\mathfrak{a}$ in the type-agent representation 
of the economy associated to an allocation rule $a$ in the original economy is defined as follows: $\mathfrak{a}_{(i, \mathcal{E})}(\omega)=a_{i}(\omega)$ if $\omega \in \mathcal{E}$ and $\mathfrak{a}_{(i, \mathcal{E})}(\omega)=0$ if $\omega \in \Omega \backslash \mathcal{E}$, for each $(i, \mathcal{E}) \in \mathcal{N}$. With this interpretation in mind, it is easy to check that the type-agent core coincides with the core as usually defined of the type-agent representation of the economy (see the appendix). Wilson (1978) proves the non-emptiness of the coarse core by checking the balancedness of a fictitious cooperative game that is closely related to the type-agent representation of the economy.

\section{Competitive Screening}

My objective is to adapt the competitive screening game of Rothschild and Stiglitz (1976) (see Mas-Colell et al., 1995, section 13.D, for a modern exposition) in order to study coalition formation. The argument extends the result of Perez-Castrillo (1994) by allowing for asymmetric information and nontransferable utility. Uninformed intermediaries (any number greater or equal to two) simultaneously offer contracts to the agents. I assume for simplicity that each intermediary proposes exactly one contract to each agent. A contract for agent $i$ is a function $c_{i}: \Omega \rightarrow \mathbb{R}^{L}$. It specifies net trades. The agents simultaneously choose at most one contract among those offered. Time goes by, uncertainty is resolved and agreed-upon contracts are realized. Suppose that agent $i$ chose some contract $c_{i}$ offered by some intermediary $j$. Let $l \in L$ and let $\omega \in \Omega$ be the state of the economy. Then agent $i$ is entitled to receive $c_{i}^{l}(\omega)$ units of good $l$ from intermediary $j$ when $c_{i}^{l}(\omega)$ is positive and intermediary $j$ is entitled to receive $-c_{i}^{l}(\omega)$ units of good $l$ from agent $i$ when $c_{i}^{l}(\omega)$ is negative.

Notice that bankruptcy is possible a priori. An intermediary could promise to deliver more goods than what he will receive from his clients. An agent could promise to deliver more goods than what he owns. I want to keep the analysis as simple as possible and be consistent with the idea underlying the core that the status quo payoffs are guaranteed when challenged. Hence I focus on markets that are regulated as follows. The regulating authority covers the deficits. At the same time, it prevents fraudulent promises by inflicting a high punishment (e.g. jail sentence or interdiction to participate to the market in the future) on the agents and the intermediaries that bankrupt. I further discuss this assumption and its consequences at the end of the section.

The preferences of the intermediaries are assumed to be continuous and strongly increasing on $\mathbb{R}_{+}^{\Omega \times L}$. In addition, each intermediary prefers having no good in each state rather than bankrupting in some state, whatever the amount of goods he receives in the other states. Similarly, each agent prefers to sign no contract rather than bankrupting in some state, whatever the amount of goods 
he receives in the other states.

A strategy for an intermediary consists in proposing a contract to each agent. A strategy for an agent is the choice of whether to sign a contract and, if so, which one, as a function of the offers made by the intermediaries. A subgame perfect equilibrium is a profile of strategies, one for each agent and each intermediary, such that 1) the choice of each agent is optimal ${ }^{2}$ in each contract signing game (even those out of the equilibrium path) and 2) the strategy of each intermediary is optimal against the strategies of the other intermediaries and the strategies of the agents. Each strategy profile generates an outcome which specifies an allocation rule for the agents, the amount of goods kept by the intermediaries, as well as the set of agents and the set of intermediaries that bankrupt, in each state of the economy.

Theorem 1 An outcome is supported by some subgame perfect equilibrium if and only if there is no bankruptcy in any state of the economy, the intermediaries exactly break even in each good in each state of the economy, and the allocation rule for the agents belongs to the type-agent core.

Proof: I consider the case where only two intermediaries are competing. The argument can easily be adapted to show that the theorem remains valid for any number of intermediaries greater or equal to two.

$\Rightarrow$ ) It is impossible that some agent bankrupts in some good in some state of the economy, as choosing to sign no contract would then be a profitable deviation. It is also impossible that some intermediary bankrupts in some good in some state of the economy as offering the null contract would then be a profitable deviation. Let $c=\left(c_{i}\right)_{i \in N}$ be the profile of contracts that is supported by the subgame perfect equilibrium. It is not necessarily directly offered by one of the two intermediaries. It is the outcome of the game following the equilibrium contract offers and selections. Let $a$ be the allocation rule associated to $c$, i.e. $a=c+e$. I prove that $a$ belongs to the type-agent core in three steps. First, $a$ is feasible. Indeed, $\sum_{i \in N} c_{i}(\omega) \leq 0$ for each $\omega \in \Omega$ as otherwise at least one of the two intermediaries bankrupts for some good in some state of the economy. Second, both intermediaries exactly break even in each good in each state of the economy. As argued before, none of the two intermediaries bankrupt in any good in any state of the economy. Hence it is sufficient to prove that $\sum_{i \in N} c_{i}(\omega) \geq 0$ for each $\omega \in \Omega$, as a positive net quantity of some good in some state of the economy for some intermediary then means a negative net quantity (bankruptcy) of that good in that state of the economy for the other intermediary. Suppose on the contrary that $\sum_{i \in N} c_{i}^{l}(\omega)<0$ for some $\omega \in \Omega$ and some $l \in L$. Then, one may construct for each $\epsilon>0$ a profile of contracts $d$ such

$\overline{2}$ As the regulating authority covers the deficits, the contract signing game is a juxtaposition of individual decision problems. The choice of an optimal contract for an agent is independent of the strategies played by the other agents. 
that the continuation payoff in good $l$ for the intermediary proposing $d$ is at least 0 when $\sum_{i \in N} c_{i}^{l}(\omega)=0$ and equals $-\sum_{i \in N} c_{i}^{l}(\omega)-\epsilon$ when $\sum_{i \in N} c_{i}^{l}(\omega)<0$, for each $\omega \in \Omega$. For sure one of the two intermediaries strictly prefers to get the aggregate equilibrium payoff rather than his own equilibrium payoff. By continuity of the preferences, proposing $d$ is a profitable deviation for at least one of the two intermediaries for each $\epsilon>0$ small enough. I now sketch how $d$ may be defined. The idea is to equally distribute to the agents in each state of the economy $\epsilon$ additional units of each good in excess supply. Formally, $d_{i}^{l}(\omega):=c_{i}^{l}(\omega)+\epsilon / n$ for each $(i, l, \omega) \in N \times L \times \Omega$ such that $\sum_{j \in N} c_{j}^{l}(\omega)<0$ and $d_{i}^{l}(\omega):=c_{i}^{l}(\omega)$ for each other triple $(i, l, \omega)$. The payoff in each good $l$ of an intermediary offering such a deviating contract indeed equals $-\sum_{i \in N} c_{i}^{l}(\omega)-\epsilon$ when $\sum_{i \in N} c_{i}^{l}(\omega)<0$, for each $\omega \in \Omega$. Nevertheless, he could possibly bankrupt in states $\omega$ such that $\sum_{i \in N} c_{i}^{l}(\omega)=0$ for each $l \in L$ as indifferent agents do not necessarily choose the deviating offer. I slightly modify the definition of $d$ at those states by making transfers between the agents. I focus on states $\omega$ such that $\sum_{i \in D(c+e, d+e, \omega)} d_{i}^{l}(\omega)>0$ for some $l \in L$. Let $i \in D(c+e, d+e, \omega)$ be such that $d_{i}^{l}(\omega)>0$. A small amount of good $l$ is transferred from agent $i$ to the other agents. Hence every agent chooses the deviating contract should it be proposed and should the future state of the economy be $\omega$. The deviating intermediary now exactly breaks even in that state as well. There could be new states where the intermediary bankrupts. Fortunately, applying the procedure recursively (using the fact that $\Omega$ is finite), I find a contract $d$ such that $\sum_{i \in D(c+e, d+e, \omega)} d_{i}^{l}(\omega) \leq 0$ for each $(l, \omega) \in L \times \Omega$. Notice though that this non positive number is not necessarily the continuation payoff of the deviating intermediary, as some agents not in $D(c+e, d+e, \omega)$ could also choose his contract should the future state be $\omega$. Therefore I modify one more time the definition of $d$ by imposing that $d_{i}(\omega)=0$ for each $\omega \in \Omega$ and each $i \in N$ such that $i \notin D(c+e, d+e, \omega)$. The above modifications do not affect the definition of $d$ at states $\omega$ such that $D(c+e, d+e, \omega)=N$. So, the payoff in each good $l$ of an intermediary offering such a deviating contract remains equal to $-\sum_{i \in N} c_{i}^{l}(\omega)-\epsilon$ when $\sum_{i \in N} c_{i}^{l}(\omega)<0$, for each $\omega \in \Omega$. Third, a is not blocked. Suppose on the contrary that $a$ is blocked by some allocation rule $a^{\prime}$. Let $c^{\prime}$ be the profile of contracts defined as follows: $c_{i}^{\prime}(\omega):=a_{i}^{\prime}(\omega)-e_{i}(\omega)$ when $i \in D\left(a, a^{\prime}, \omega\right)$ and $c_{i}^{\prime}(\omega):=0$ when $i \in N \backslash D\left(a, a^{\prime}, \omega\right)$, for each $\omega \in \Omega$. The intermediary proposing $c^{\prime}$ breaks even in each state of the economy and keeps some strictly positive amount of some goods in some states of the economy. Hence $c^{\prime}$ is a profitable deviation for both intermediaries, given the second step of the proof.

$\Leftarrow)$ Let $a$ be a feasible allocation rule that cannot be blocked by any allocation rule. I consider the following strategies. Both intermediaries propose $c=a-e$. All the agents go to the first intermediary. If the first intermediary proposes something different from $c$, then each agent chooses to stay with him if and only if he strictly prefers his proposal to $c$. Otherwise the agents go to the second intermediary. If the second intermediary proposes something different 
from $c$, then each agent chooses to follow him if and only if he strictly prefers his proposal to $c$. Otherwise the agents stay with the first intermediary. These strategies are clearly part of a subgame perfect equilibrium.

There is no communication and no information transmission. First, the agents do not deduce any information from observing the alternative contracts as the intermediaries are uninformed. Second, the agents do not learn any information at the contract signing stage as they sign the contracts simultaneously. Nevertheless, I obtain a refinement of the coarse core (see theorem 2 and example 1 hereafter) because I apply a screening argument to coalition formation.

Some outcomes of the game are not feasible if the regulating authority has no goods at its disposal. Even if the intermediaries break even in each good in each state of the economy at equilibrium and even if the intermediaries break even in each good in each state of the economy when proposing a deviating contract, it may be the case that some of the active intermediaries facing an objection bankrupt. This is the usual feature of the competitive screening games. In Rothschild and Stiglitz (1976), the pooling contract is subject to a separating objection attracting only the low risk agents when they are not too numerous in the population. The insurance company offering the pooling contract bankrupts as it remains with the high risk agents.

If the regulating authority does not have goods at its disposal for covering the deficits, and if the agents are fully rational in the sense that they anticipate the possibility of bankruptcy, then one faces an important conceptual difficulty. Suppose that each intermediary has to specify in each state of the economy how he will re-allocate the endowment of its clients as a function of the set of agents that choose him. Then the contract signing stage is a Bayesian game instead of a juxtaposition of independent individual decision problems. Any allocation rule that is interim individually rational is supported by some subgame perfect Bayesian equilibrium of the competitive screening game. Indeed, choosing an alternative contract is never profitable if nobody else is signing it. There is a problem of coordination and of equilibrium selection.

I conclude the section by pointing out some differences with respect to the model of Rothschild and Stiglitz. First, I assume that the information of the agents is verifiable at the time of implementing the contracts, although the risk profile of the agents is not observable when the contract is realized in their model. Second, my argument is not restricted to one good (money). Third, feasibility is not expressed in expected terms. The intermediaries break even in each good in each state of the economy at equilibrium. 


\section{$5 \quad$ Properties}

Let $S$ be a coalition. An event $\mathcal{E} \subseteq \Omega$ is common knowledge among the members of $S$ if it can be written as a union of elements of $\mathcal{P}_{i}$ for each $i \in S$. An allocation rule $a$ is feasible for $S$ if $\sum_{i \in S} a_{i}(\omega) \leq \sum_{i \in S} e_{i}(\omega)$ for each $\omega \in \Omega$. Coalition $S$ has a coarse objection against an allocation rule $a$ if there exist an allocation rule $a^{\prime}$ feasible for $S$ and an event $\mathcal{E}$ that is common knowledge among the members of $S$ such that $E\left(u_{i}\left(a_{i}^{\prime}\right) \mid P_{i}(\omega)\right)>E\left(u_{i}\left(a_{i}\right) \mid P_{i}(\omega)\right)$ for each $i \in S$ and each $\omega \in \mathcal{E}$. The coarse core is the set of feasible allocation rules against which no coalition has a coarse objection (Wilson, 1978).

Theorem 2 The type-agent core is a subset of the coarse core.

Proof: Let $a$ be an allocation rule and let $\left(S, a^{\prime}, \mathcal{E}\right)$ be a coarse objection against $a$. Then $a$ is blocked by the allocation rule $a^{\prime \prime}$ where $a_{i}^{\prime \prime}(\omega):=(1-\epsilon) a_{i}^{\prime}(\omega)$ if $(i, \omega) \in S \times \mathcal{E}$ and $a_{i}^{\prime \prime}(\omega):=e_{i}(\omega)$ otherwise $(\epsilon>0$ is very small $)$.

Let $a$ be an allocation rule. A coalition $S$ has a fine objection against $a$ if there exist an event $\mathcal{E}$ and an allocation rule $a^{\prime}$ feasible for $S$ such that the two following properties are true at each $\left.\omega \in \mathcal{E}: 1) \bigcap_{i \in S} P_{i}(\omega) \subseteq \mathcal{E} ; 2\right) E\left(u_{i}\left(a_{i}^{\prime}\right) \mid \mathcal{E} \cap\right.$ $\left.P_{i}(\omega)\right)>E\left(u_{i}\left(a_{i}\right) \mid \mathcal{E} \cap P_{i}(\omega)\right)$ for each $i \in S$. The fine core is the set of feasible allocation rules against which no coalition has a fine objection (Wilson, 1978). The fine core is a subset of the coarse core. In fact, the fine core is the smallest conceivable core according to Wilson, as fine objections allow for any kind of information sharing. This is wrong once we try to understand how the agreements emerge instead of testing given allocation rules. Example 11 in de Clippel and Minelli (2003) illustrates this point when the tentative agreements are proposed by the agents themselves. I adapt the example in order to show that some fine core allocations may be blocked.

Example 1 I consider a sunspot economy with asymmetric information. There are two agents, two goods and two equiprobable states for the economy. Agent 1 knows the future state while agent 2 does not: $\mathcal{P}_{1}=\left\{\left\{\omega_{1}\right\},\left\{\omega_{2}\right\}\right\}$ and $\mathcal{P}_{2}=\left\{\left\{\omega_{1}, \omega_{2}\right\}\right\}$. The endowments are defined as follows: $e_{1}(\omega)=(0,100)$ and $e_{2}(\omega)=(1,100)$ for each $\omega \in \Omega$. The utility functions are defined as follows: $u_{1}(x, \omega)=100 x^{1}+x^{2}-100$ and $u_{2}(x, \omega)=x^{1}+x^{2}-101$ for each $x \in \mathbb{R}_{+}^{2}$ and each $\omega \in\left\{\omega_{1}, \omega_{2}\right\}$. It is mutually beneficial to exchange good 1. Good 2 is money. Consider the two following feasible allocation rules.

\begin{tabular}{c|cc|cc} 
& $a_{1}$ & $a_{2}$ & $a_{1}^{\prime}$ & $a_{2}^{\prime}$ \\
\hline$\omega_{1}$ & $(1,198)$ & $(0,2)$ & $(0,100)$ & $(1,100)$ \\
$\omega_{2}$ & $(1,0)$ & $(0,200)$ & $(1,97)$ & $(0,102)$
\end{tabular}


The allocation rule $a$ belongs to the fine core but not to the type-agent core. Indeed, it favors too much agent 1 in $\omega_{1}$ and hence is blocked, by $a^{\prime}$ for instance.

There is no general inclusion relation between the type-agent core and the fine core. Here is an example where the fine core is a subset of the type-agent core.

Example 2 I adapt example 2 of Wilson (1978). There are three agents, one good (money) and two equiprobable state for the economy. Agent 3 knows the future state while agents 1 and 2 do not: $\mathcal{P}_{1}=\mathcal{P}_{2}=\left\{\omega_{1}, \omega_{2}\right\}$ and $\mathcal{P}_{3}=$ $\left\{\left\{\omega_{1}\right\},\left\{\omega_{2}\right\}\right\}$. The endowments are defined as follows: $e\left(\omega_{1}\right)=(100,0,0)$ and $e\left(\omega_{2}\right)=(0,100,0)$. The utility functions are defined as follows: $u_{i}(x, \omega)=\sqrt{x}$ for each $i \in\{1,2,3\}$, each $x \in \mathbb{R}_{+}$and each $\omega \in\left\{\omega_{1}, \omega_{2}\right\}$. The full-insurance allocation rule giving 50 dollars to each of the two first agents in each state of the economy belongs to both the type-agent core and the coarse core but not to the fine core. If the agents can communicate, then agent 3 will meet agent 1 when the state is $\omega_{1}$, convince him that the future state is favorable to him, and agree with him to implement a different allocation, for instance $(75,0,25)$.

Notice that the fine core may be empty, as in the previous example. Hence it is remarkable that the type-agent core is never empty even if it is included in the coarse core and may sometimes be a subset of the fine core.

Theorem 3 The type-agent core is not empty.

The result is a consequence of Scarf (1967), as the type-agent core coincides with the core of the type-agent representation of the economy.

\section{Convergence}

An allocation rule $a$ is a constrained market equilibrium if it is feasible and there exists a price system $p: \Omega \rightarrow \mathbb{R}_{+}^{L}$ such that

$$
E\left(u_{i}\left(a_{i}^{\prime}\right) \mid P_{i}(\omega)\right) \leq E\left(u_{i}\left(a_{i}\right) \mid P_{i}(\omega)\right)
$$

for each $a_{i}^{\prime} \in \mathbb{R}_{+}^{L \times P_{i}(\omega)}$ with $\sum_{\omega^{\prime} \in P_{i}(\omega)} p\left(\omega^{\prime}\right) \cdot a_{i}^{\prime}\left(\omega^{\prime}\right) \leq \sum_{\omega^{\prime} \in P_{i}(\omega)} p\left(\omega^{\prime}\right) \cdot e_{i}\left(\omega^{\prime}\right)$, each $\omega \in \Omega$ and each $i \in N$ (Wilson, 1978, footnote 6 ). It is a natural generalization of the Arrow-Debreu equilibrium in markets with contingent commodities to economies with asymmetric information when inside trading is prohibited. Indeed, in a world with contingent commodities, the uninformed invisible hand' specifies a price for each commodity in each state of the economy in order to clear all the markets. The agents do not learn anything by observing the price vector as it does not depend on the future state of the economy. They maximize their expected utilities under the additional constraint that 
they may not sell contingent commodities associated to states that they know are not going to occur. I further analyze example 2 of Wilson (1978) in order to illustrate the concept.

Example 3 There are three agents, one good (money) and three equiprobable states for the economy. The following table specifies the information and the endowments of the agents.

\begin{tabular}{c|c|ccc}
$\operatorname{Agent}(i)$ & $\mathcal{P}_{i}$ & $e_{i}\left(\omega_{1}\right)$ & $e_{i}\left(\omega_{2}\right)$ & $e_{i}\left(\omega_{3}\right)$ \\
\hline 1 & $\left\{\left\{\omega_{1}\right\},\left\{\omega_{2}, \omega_{3}\right\}\right\}$ & 5 & 1 & 3 \\
2 & $\left\{\left\{\omega_{2}\right\},\left\{\omega_{1}, \omega_{3}\right\}\right\}$ & 3 & 5 & 1 \\
3 & $\left\{\left\{\omega_{3}\right\},\left\{\omega_{1}, \omega_{2}\right\}\right\}$ & 1 & 3 & 5
\end{tabular}

The following table specifies an allocation rule $a$ that is a constrained market equilibrium for the price vector $(1,1,1)$. It also specifies the associated nettrades.

\begin{tabular}{c|ccc|ccc} 
Agent $(i)$ & $a_{i}\left(\omega_{1}\right)$ & $a_{i}\left(\omega_{1}\right)$ & $a_{i}\left(\omega_{1}\right)$ & $z_{i}\left(\omega_{1}\right)$ & $z_{i}\left(\omega_{2}\right)$ & $z_{i}\left(\omega_{3}\right)$ \\
\hline 1 & 5 & 2 & 2 & 0 & 1 & -1 \\
2 & 2 & 5 & 2 & -1 & 0 & 1 \\
3 & 2 & 2 & 5 & 1 & -1 & 0
\end{tabular}

The equilibrium concept allows for some insurance between the two agents that are not fully informed. This would be impossible if the prices of the three contingent commodities were varying with the future state of the economy, much as in rational expectations equilibria. Markets have to clear ex-post with Wilson's concept. If for instance the future state is $\omega_{1}$, there is excess supply (resp. demand) of money in state 2 (resp. 3), as agent 1 is prohibited to buy or sell these contingent commodities. This is irrelevant as these claims will not have to be satisfied. What matters on the other hand is the fact that the demand for money from agent 3 is met by the supply of money by agent 2 in state 1 . Varying the states, there are three equilibrium equations to be satisfied in total, not nine. The market is not fully decentralized in that sense. I think instead of an uninformed trading organization proposing the prices in order to match demand with supply ex-post.

It is easy to check that the set of constrained market equilibria coincides with the set of Arrow-Debreu equilibria of the type-agent representation of the 
economy suggested in section 3 . Hence the next three theorems are corollaries ${ }^{3}$ of Debreu and Scarf (1963) (see the appendix).

Theorem 4 Suppose that each agent is endowed with a strictly positive amount of each good in each state of the economy. Then the set of constrained market equilibria is not empty.

Theorem 5 The set of constrained market equilibria is a subset of the typeagent core.

I replicate the agents of the economy described in section 2 as in Serrano et al. (2001). Let $\bar{k}$ be the number of replicas. Each agent of the original economy now appears $\bar{k}$ times. Hence, there are $\bar{k} N$ agents in the replicated economy. Let $i \in N$ and let $k \in\{1, \ldots, \bar{k}\}$. Copy $k$ of agent $i$ is denoted $i . k$. Agent $i . k$ in the replicated economy has the same endowment, the same information and the same utility function as agent $i$ in the original economy.

Theorem 6 Suppose that each agent's utility function is strictly concave in each state of the economy and that each agent is endowed with a strictly positive amount of each good in each state of the economy. Then, the type-agent core shrinks to the set of constrained market equilibria as the number of replicas $\bar{k}$ tends to infinity. Observe that the type-agent representation of the replicated economy coincides with the Debreu-Scarf replication of the type-agent representation of the original economy.

A similar result is obtained by Goenka and Shell (1997) for sunspot economies with restricted market participation. There is uncertainty but no asymmetric information. The uncertainty does not affect the fundamentals of the economy. The agents are not necessarily allowed to trade on each market for contingent goods. Even if the interpretation of the models differ, the notion of sunspot equilibrium formally coincides with the notion of constrained market equilibrium and the idea of quasi-Walrasian economy (Balasko et al., 1995) is equivalent to the type-agent representation I suggest for exchange economies with asymmetric information. Goenka and Shell define the core of the sunspot economy with restricted market participation as the core of the quasi-Walrasian economy. Without justifying the definition, they prove a similar convergence result (Goenka and Shell, 1997, theorem 7.3; Serrano et al., 2001, section 4.1). It is not clear whether there is anything more than a formal relation between sunspot economies with restricted market participation and economies with asymmetric information. In any case, the former would constitute only a small subclass of the latter.

3 The assumptions of Debreu and Scarf (1963, section 5) are satisfied if the consumption set of type-agent $(i, \mathcal{E})$ is the set of contingent goods that are compatible with his information, i.e. $\mathbb{R}_{+}^{L \times \mathcal{E}}$, for each $(i, \mathcal{E}) \in \mathcal{N}$. 
Serrano et al. (2001) argue on a simple example that there is no hope to obtain a convergence result for the coarse or the fine core towards constrained market equilibria. They show that the negative result is robust with respect to many alternative definitions of both the core and the price equilibria. I now explain why their argument does not apply to the type-agent core.

Example 4 Consider a sunspot economy with two agents, two goods and two equiprobable states for the economy. Agent 1 knows the future state while agent 2 does not: $\mathcal{P}_{1}=\left\{\left\{\omega_{1}\right\},\left\{\omega_{2}\right\}\right\}$ and $\mathcal{P}_{2}=\left\{\left\{\omega_{1}, \omega_{2}\right\}\right\}$. The endowments are defined as follows: $e_{1}(\omega)=(24,0)$ and $e_{2}(\omega)=(0,24)$ for each $\omega \in \Omega$. The utility functions are defined as follows: $u_{1}(x, \omega)=u_{2}(x, \omega)=\sqrt{x^{1} x^{2}}$ for each $x \in \mathbb{R}_{+}^{2}$ and each $\omega \in\left\{\omega_{1}, \omega_{2}\right\}$. Let $a$ be the feasible allocation rule defined as follows: $a_{1}\left(\omega_{1}\right):=(15,15), a_{1}\left(\omega_{2}\right):=(8,8), a_{2}\left(\omega_{1}\right):=(9,9)$ and $a_{2}\left(\omega_{2}\right):=(16,16)$. Serrano et al. show that the $\bar{k}$-replication of $a$ belongs to the coarse core but is not a constrained market equilibrium of the $\bar{k}$-replicated economy, for each $\bar{k} \in \mathbb{N}$. It is already blocked in the second replica. The second replication of $a$ is given by:

\begin{tabular}{c|cccc}
$a^{\prime}$ & 1.1 & 1.2 & 2.1 & 2.2 \\
\hline$\omega_{1}$ & $(15,15)$ & $(15,15)$ & $(9,9)$ & $(9,9)$ \\
$\omega_{2}$ & $(8,8)$ & $(8,8)$ & $(16,16)$ & $(16,16)$
\end{tabular}

It is blocked by the following allocation rule:

\begin{tabular}{c|cccc}
$a^{\prime \prime}$ & 1.1 & 1.2 & 2.1 & 2.2 \\
\hline$\omega_{1}$ & $(31 / 2,31 / 2)$ & $(0,0)$ & $(17 / 2,17 / 2)$ & $(0,0)$ \\
$\omega_{2}$ & $(10,13 / 2)$ & $(10,13 / 2)$ & $(28,11)$ & $(0,0)$
\end{tabular}

The coalitions are forming as follows: $\{1.1,2.1\}$ if the future state is $\omega_{1}$ and $\{1.1,1.2,2.1\}$ if the future state is $\omega_{2}$.

\section{Conclusion}

I studied exchange economies with asymmetric information, assuming that cooperation is achieved through the help of uninformed intermediaries that compete in an attempt to make some profit. I defined a new notion of core that coincides with the subgame perfect equilibrium outcomes of some competitive screening game. Contrarily to Wilson (1978)'s analysis, objections 
are not bound to emerge from state-independent coalitions because the intermediaries anticipate each agent's participation decision as a function of its private information. The coalitions that form are endogenously determined by comparing different allocation rules. This explains why the type-agent core may be a strict subset of the coarse core even though it involves no communication and no information transmission. I proved that the type-agent core converges towards the set of constrained market equilibria when the economy is replicated.

I suggest some directions for further research. Communication and information transmission could be discussed by modifying the competitive screening game. The intermediaries could be partially informed and learn additional information by observing the choices of the agents. The agents themselves could learn some information before choosing a contract if they observe the choice of other agents. More generally, there is a need for a better strategic foundation of core concepts in exchange economies with asymmetric information. ${ }^{4}$ I analyzed a variant of the procedure suggested by Perez-Castrillo (1994). Other procedures supporting the core under complete information (see e.g. Perry and Reny, 1994; Serrano, 1995; Serrano and Vohra, 1997) should be studied as well. Finally, the type-agent core and its non-cooperative justification should be extended to situations where the information is not verifiable at the time of implementing the agreements. Myerson (1995, 2003) considers entrepreneurs devising alternative market organizations and mediators helping the agents to coordinate. It would be illuminating if his core concepts could be characterized as the set of equilibrium outcomes associated with some explicit competitive screening game.

\section{References}

[1] Balasko, Y., D. Cass and K. Shell, 1995. Market Participation and Sunspot Equilibria. Review of Economic Studies 62, 491-512.

[2] Debreu, G. and H. Scarf, 1963. A Limit Theorem on the Core of an Economy. International Economic Review 4, 235-246.

[3] de Clippel, G. and E. Minelli, 2003. Two-Person Bargaining with Verifiable Information. forthcoming Journal of Mathematical Economics.

[4] Goenka, A. and K. Shell, 1997. Robustness of Sunspot Equilibria. Economic Theory 10, 79-98.

\footnotetext{
${ }_{4}$ Serrano and Vohra (2004) is another paper going in that direction. They characterize the set of allocation rules that are robust to coalitional deviations that require the unanimous support of its members in some explicit voting games.
} 
[5] Harsanyi, J. C., 1967-68. Games with Incomplete Information Played by Bayesian Players. Management Science 14, 159-182, 320-334, 486-502.

[6] Mas-Colell, A., M. D. Whinston and J. R. Green, 1995. Microeconomic Theory. Oxford University Press.

[7] Myerson, R. B., 1995. Sustainable Matching Plans with Adverse Selection. Games and Economic Behavior 9, 35-65.

[8] Myerson, R. B., 2003. Virtual Utility and the Core for Games. Mimeo, University of Chicago.

[9] Perez-Castrillo, D., 1994. Cooperative Outcomes through Noncooperative Games. Games and Economic Behavior 7, 428-440.

[10] Perry, M. and P. Reny, 1994. A non-Cooperative View of Coalition Formation and the Core. Econometrica 62, 795-817.

[11] Rothschild, M. and J. Stiglitz 1976. Equilibrium in Competitive Insurance Markets: An Essay on the Economics of Imperfect Information. Quarterly Journal of Economics 90, 629-649.

[12] Scarf, H. E., 1967. The Core of an N-Person Game. Econometrica 35, 50-69.

[13] Serrano, R. 1995. A Market to Implement the Core. Journal of Economic Theory 67, 285-294.

[14] Serrano, R. and R. Vohra, 1997. Non-Cooperative Implementation of the Core. Social Choice and Welfare 14, 513-525.

[15] Serrano, R. and R. Vohra 2004. Information Transmission in Coalitional Voting Games. Mimeo, Brown University.

[16] Serrano, R., R. Vohra and O. Volij, 2001. On the Failure of Core Convergence in Economies with Asymmetric Information. Econometrica 69, 1685-1696.

[17] Wilson, R., 1978. Information, Efficiency, and the Core of an Economy. Econometrica 46, 807-816. 
Appendix The Core and the Arrow-Debreu Equilibria of the Type-Agent Representation

I refer to the last paragraph of section 3 for the definition of the type-agent representation of the economy. I denote by $\mathfrak{e}_{(i, \mathcal{E})}: \Omega \rightarrow \mathbb{R}_{+}^{L}$ the endowment of type-agent $(i, \mathcal{E}): \mathfrak{e}_{(i, \mathcal{E})}(\omega):=e_{i}(\omega)$ if $\omega \in \mathcal{E}, \mathfrak{e}_{(i, \mathcal{E})}(\omega):=0$ if $\omega \in \Omega \backslash \mathcal{E}$, for each $(i, \mathcal{E}) \in \mathcal{N}$. Subsets of $\mathcal{N}$ are called coalitions. An allocation rule $\mathfrak{a}$ is feasible for coalition $\mathbb{S}$ if $\sum_{(i, \mathcal{E}) \in \mathbb{S}} \mathfrak{a}_{(i, \mathcal{E})}(\omega) \leq \sum_{(i, \mathcal{E}) \in \mathbb{S}} \mathfrak{e}_{(i, \mathcal{E})}(\omega)$ for each $\omega \in \Omega$. An allocation rule $\mathfrak{a}^{\prime}$ Pareto dominates an allocation rule $\mathfrak{a}$ for coalition $\mathbb{S}$ if $E\left(u_{(i, \mathcal{E})}\left(\mathfrak{a}_{(i, \mathcal{E})}^{\prime}\right)\right)>E\left(u_{(i, \mathcal{E})}\left(\mathfrak{a}_{(i, \mathcal{E})}\right)\right)$ for each $(i, \mathcal{E}) \in \mathbb{S}$. The core is the set of allocation rules that are feasible for $\mathcal{N}$ and such that there do not exist a coalition $\mathbb{S}$ and an allocation rule $\mathfrak{a}^{\prime}$ feasible for $\mathbb{S}$ that Pareto dominates $\mathfrak{a}$ for $\mathbb{S}$.

Proposition A1 Let a be an allocation rule that belongs to the type-agent core. Then the associated allocation rule $\mathfrak{a}$ belongs to the core of the type-agent representation of the economy.

Proof: Notice that $\mathfrak{a}$ is feasible for $\mathcal{N}$. Indeed, for each $\omega \in \Omega, \sum_{i \in N} a_{i}(\omega) \leq$ $\sum_{i \in N} e_{i}(\omega)$ implies that $\sum_{i \in N} \mathfrak{a}_{\left(i, P_{i}(\omega)\right)}(\omega) \leq \sum_{i \in N} \mathfrak{e}_{\left(i, P_{i}(\omega)\right)}(\omega)$ which, in turn, implies that $\sum_{(i, \mathcal{E}) \in \mathcal{N}} \mathfrak{a}_{(i, \mathcal{E})}(\omega) \leq \sum_{(i, \mathcal{E}) \in \mathcal{N}} \mathfrak{e}_{(i, \mathcal{E})}(\omega)$. Suppose now that there exist a coalition $\mathbb{S}$ and an allocation rule $\mathfrak{a}^{\prime}$ feasible for $\mathbb{S}$ that Pareto dominates $\mathfrak{a}$ for $\mathbb{S}$ in the type-agent representation of the economy. Given the preferences of the typeagents, I may assume without loss of generality that $\mathfrak{a}_{(i, \mathcal{E})}^{\prime}(\omega)=0$ for each $(i, \mathcal{E}) \in \mathcal{N}$ and each $\omega \in \Omega$ such that $\omega \notin \mathcal{E}$. Let then $a^{\prime}$ be the allocation rule defined as follows: $a_{i}^{\prime}(\omega):=\mathfrak{a}_{\left(i, P_{i}(\omega)\right)}^{\prime}(\omega)$ for each couple $(i, \omega) \in N \times \Omega$ such that $\left(i, P_{i}(\omega)\right) \in \mathbb{S}$, and $a_{i}^{\prime}(\omega):=0$ for each other couple $(i, \omega) \in N \times \Omega$. Notice that, for each $\omega \in \Omega$, $i \in D\left(a, a^{\prime}, \omega\right)$ if and only if $\left(i, P_{i}(\omega)\right) \in \mathbb{S}$. We have:

$$
\begin{aligned}
\sum_{i \in D\left(a, a^{\prime}, \omega\right)} a_{i}^{\prime}(\omega) & =\sum_{i \in N \text { s.t. }\left(i, P_{i}(\omega)\right) \in \mathbb{S}} a_{i}^{\prime}(\omega) \\
& =\sum_{i \in N \text { s.t. }\left(i, P_{i}(\omega)\right) \in \mathbb{S}} \mathfrak{a}_{\left(i, P_{i}(\omega)\right)}^{\prime}(\omega) \\
& =\sum_{(i, \mathcal{E}) \in \mathbb{S}} \mathfrak{a}_{(i, \mathcal{E})}^{\prime}(\omega) \\
& \leq \sum_{(i, \mathcal{E}) \in \mathbb{S}} \mathfrak{e}_{(i, \mathcal{E})}(\omega) \\
& =\sum_{i \in N} \text { s.t. }\left(i, P_{i}(\omega)\right) \in \mathbb{S} \mathfrak{e}_{\left(i, P_{i}(\omega)\right)}(\omega) \\
& =\sum_{i \in N} \text { s.t. }\left(i, P_{i}(\omega)\right) \in \mathbb{S} \\
& e_{i}(\omega) \\
& =\sum_{i \in D\left(a, a^{\prime}, \omega\right)} e_{i}(\omega),
\end{aligned}
$$

for each $\omega \in \Omega$. If $a^{\prime}$ is not strictly feasible when proposed against $a$, then one may slightly modify $a^{\prime}$ as follows. There exist $\omega \in \Omega$ and $i \in D\left(a, a^{\prime}, \omega\right)$ such that $a_{i}^{\prime}(\omega)>$ 0 . One of the positive components of $a_{i}^{\prime}(\omega)$ is decreased while keeping $D\left(a, a^{\prime}, \omega\right)$ unchanged. The resulting allocation rule is strictly feasible when proposed against $a$.

Proposition A2 Let $\mathfrak{a}$ be an allocation rule that belongs to the core of the typeagent representation of the economy. Then the associated allocation rule a belongs to the type-agent core.

Proof: a) Notice that $a$ is feasible. Indeed, for each $\omega \in \Omega, \sum_{(i, \mathcal{E}) \in \mathcal{N}} \mathfrak{a}_{(i, \mathcal{E})}(\omega) \leq$ 
$\sum_{(i, \mathcal{E}) \in \mathcal{N}^{\mathfrak{e}}(i, \mathcal{E})}(\omega)$ implies that $\sum_{i \in N} \mathfrak{a}_{\left(i, P_{i}(\omega)\right)}(\omega) \leq \sum_{i \in N} \mathfrak{e}_{\left(i, P_{i}(\omega)\right)}(\omega)$ which, in turn, implies that $\sum_{i \in N} a_{i}(\omega) \leq \sum_{i \in N} e_{i}(\omega)$. Suppose now that $a$ is blocked by an allocation rule $a^{\prime}$ in the original economy. Let then $\mathbb{S}$ be the coalition defined as follows: $\mathbb{S}=\left\{(i, \mathcal{E}) \in \mathcal{N} \mid E\left(u_{i}\left(a_{i}^{\prime}\right) \mid \mathcal{E}\right)>E\left(u_{i}\left(a_{i}\right) \mid \mathcal{E}\right)\right\}$. Let also $\mathfrak{a}^{\prime}$ be an allocation rule such that $\mathfrak{a}_{(i, \mathcal{E})}^{\prime}(\omega)=a_{i}^{\prime}(\omega)$ if $\omega \in \mathcal{E}$ and $\mathfrak{a}_{(i, \mathcal{E})}^{\prime}(\omega)=0$ if $\omega \in \Omega \backslash \mathcal{E}$, for each each $(i, \mathcal{E}) \in \mathbb{S}$. Observe that $\mathfrak{a}^{\prime}$ is feasible for $\mathbb{S}$. Indeed, we have:

$$
\begin{aligned}
\sum_{(i, \mathcal{E}) \in \mathbb{S}} \mathfrak{a}_{(i, \mathcal{E})}^{\prime}(\omega) & =\sum_{(i, \mathcal{E}) \in \mathbb{S} \text { s.t. } \omega \in \mathcal{E}} a_{i}^{\prime}(\omega) \\
& =\sum_{i \in D\left(a, a^{\prime}, \omega\right)} a_{i}^{\prime}(\omega) \\
& \leq \sum_{i \in D\left(a, a^{\prime}, \omega\right)} e_{i}(\omega) \\
& =\sum_{(i, \mathcal{E}) \in \mathbb{S} \text { s.t. } \omega \in \mathcal{E}} e_{i}(\omega) \\
& =\sum_{(i, \mathcal{E}) \in \mathbb{S}} \mathfrak{e}_{(i, \mathcal{E})}(\omega)
\end{aligned}
$$

for each $\omega \in \Omega$. Observe also that $\mathfrak{a}^{\prime}$ Pareto dominates $\mathfrak{a}$ for $\mathbb{S}$. Indeed, we have:

$$
\begin{aligned}
E\left(u_{(i, \mathcal{E})}\left(\mathfrak{a}_{(i, \mathcal{E})}^{\prime}\right)\right) & =\sum_{\omega \in \mathcal{E}} \pi(\omega) u_{i}\left(a_{i}^{\prime}(\omega), \omega\right) \\
& =\pi(\mathcal{E}) E\left(u_{i}\left(a_{i}^{\prime}\right) \mid \mathcal{E}\right) \\
& >\pi(\mathcal{E}) E\left(u_{i}\left(a_{i}\right) \mid \mathcal{E}\right) \\
& =\sum_{\omega \in \mathcal{E}} \pi(\omega) u_{i}\left(a_{i}(\omega), \omega\right) \\
& =E\left(u_{(i, \mathcal{E})}\left(\mathfrak{a}_{(i, \mathcal{E})}\right)\right)
\end{aligned}
$$

for each $(i, \mathcal{E}) \in \mathbb{S}$.

An allocation rule $\mathfrak{a}$ is an Arrow-Debreu equilibrium in the type-agent representation of the economy if it is feasible for $\mathcal{N}$ and there exists a price system $p: \Omega \rightarrow \mathbb{R}_{+}^{L}$ such that

$$
E\left(u_{(i, \mathcal{E})}\left(\mathfrak{a}_{(i, \mathcal{E})}^{\prime}\right)\right) \leq E\left(u_{(i, \mathcal{E})}\left(\mathfrak{a}_{(i, \mathcal{E})}\right)\right)
$$

for each $\mathfrak{a}_{(i, \mathcal{E})}^{\prime} \in \mathbb{R}_{+}^{L \times \Omega}$ with $\sum_{\omega^{\prime} \in \Omega} p\left(\omega^{\prime}\right) \cdot \mathfrak{a}_{(i, \mathcal{E})}^{\prime}\left(\omega^{\prime}\right) \leq \sum_{\omega^{\prime} \in \Omega} p\left(\omega^{\prime}\right) \cdot \mathfrak{e}_{(i, \mathcal{E})}\left(\omega^{\prime}\right)$, and each $(i, \mathcal{E}) \in \mathcal{N}$.

Proposition B1 Let a be a constrained market equilibrium. Then the associated allocation rule $\mathfrak{a}$ is an Arrow-Debreu equilibrium in the type-agent representation of the economy.

Proof: Notice that $\mathfrak{a}$ is feasible for $\mathcal{N}$ as in proposition A1. Let $p$ be the price system supporting $a$ as a constrained market equilibrium. Let $(i, \mathcal{E}) \in \mathcal{N}$ and let $\mathfrak{a}_{(i, \mathcal{E})}^{\prime} \in \mathbb{R}_{+}^{L \times \Omega}$ be such that $\sum_{\omega^{\prime} \in \Omega} p\left(\omega^{\prime}\right) \cdot \mathfrak{a}_{(i, \mathcal{E})}^{\prime}\left(\omega^{\prime}\right) \leq \sum_{\omega^{\prime} \in \Omega} p\left(\omega^{\prime}\right) \cdot \mathfrak{e}_{(i, \mathcal{E})}\left(\omega^{\prime}\right)$. Given the preferences of type-agent $(i, \mathcal{E})$, I may assume without loss of generality that $\mathfrak{a}_{(i, \mathcal{E})}^{\prime}(\omega)=0$ for each $\omega \in \Omega \backslash \mathcal{E}$. Let $\omega \in \mathcal{E}$ and let $a_{i}^{\prime} \in \mathbb{R}_{+}^{L \times P_{i}(\omega)}$ be defined as follows: $a_{i}^{\prime}\left(\omega^{\prime}\right):=\mathfrak{a}_{\left(i, P_{i}(\omega)\right)}^{\prime}\left(\omega^{\prime}\right)$ for each $\omega^{\prime} \in P_{i}(\omega)(=\mathcal{E})$. Then,

$$
\begin{aligned}
\sum_{\omega^{\prime} \in P_{i}(\omega)} p\left(\omega^{\prime}\right) \cdot a_{i}^{\prime}\left(\omega^{\prime}\right) & =\sum_{\omega^{\prime} \in \Omega} p\left(\omega^{\prime}\right) \cdot \mathfrak{a}_{(i, \mathcal{E})}^{\prime}\left(\omega^{\prime}\right) \\
& \leq \sum_{\omega^{\prime} \in \Omega} p\left(\omega^{\prime}\right) \cdot \mathfrak{e}_{(i, \mathcal{E})}\left(\omega^{\prime}\right) \\
& =\sum_{\omega^{\prime} \in P_{i}(\omega)} p\left(\omega^{\prime}\right) \cdot e_{i}\left(\omega^{\prime}\right) .
\end{aligned}
$$

Hence, $E\left(u_{(i, \mathcal{E})}\left(\mathfrak{a}_{(i, \mathcal{E})}^{\prime}\right)\right)=\pi\left(P_{i}(\omega)\right) E\left(u_{i}\left(a_{i}^{\prime}\right) \mid P_{i}(\omega)\right) \leq \pi\left(P_{i}(\omega)\right) E\left(u_{i}\left(a_{i}\right) \mid P_{i}(\omega)\right)=$ $E\left(u_{(i, \mathcal{E})}\left(\mathfrak{a}_{(i, \mathcal{E})}\right)\right)$. 
Proposition B2 Let $\mathfrak{a}$ be an Arrow-Debreu equilibrium in the type-agent representation of the economy. Then the associated allocation rule a is a constrained market equilibrium.

Proof: Notice that $a$ is feasible as in proposition A2. Let $p$ be the price system supporting $\mathfrak{a}$ as an Arrow-Debreu equilibrium in the type-agent representation of the economy. Let $i \in N$, let $\omega \in \Omega$, let $a_{i}^{\prime} \in \mathbb{R}_{+}^{L \times P_{i}(\omega)}$ be such that $\sum_{\omega^{\prime} \in P_{i}(\omega)} p\left(\omega^{\prime}\right) \cdot a_{i}^{\prime}\left(\omega^{\prime}\right) \leq \sum_{\omega^{\prime} \in P_{i}(\omega)} p\left(\omega^{\prime}\right) \cdot e_{i}\left(\omega^{\prime}\right)$ and let $\mathfrak{a}_{i}^{\prime} \in \mathbb{R}_{+}^{L \times \Omega}$ be defined as follows: $\mathfrak{a}_{(i, \mathcal{E})}^{\prime}\left(\omega^{\prime}\right):=a_{i}^{\prime}\left(\omega^{\prime}\right)$ for each $\omega^{\prime} \in P_{i}(\omega)$ and $\mathfrak{a}_{(i, \mathcal{E})}^{\prime}\left(\omega^{\prime}\right):=0$ for each $\omega^{\prime} \in$ $\Omega \backslash P_{i}(\omega)$. Then,

$$
\begin{aligned}
\sum_{\omega^{\prime} \in \Omega} p\left(\omega^{\prime}\right) \cdot \mathfrak{a}_{\left(i, \mathcal{P}_{i}(\omega)\right)}^{\prime}\left(\omega^{\prime}\right) & =\sum_{\omega^{\prime} \in P_{i}(\omega)} p\left(\omega^{\prime}\right) \cdot a_{i}^{\prime}\left(\omega^{\prime}\right) \\
& \leq \sum_{\omega^{\prime} \in P_{i}(\omega)} p\left(\omega^{\prime}\right) \cdot e_{i}\left(\omega^{\prime}\right) \\
& =\sum_{\omega^{\prime} \in \Omega} p\left(\omega^{\prime}\right) \cdot \mathfrak{e}_{\left(i, \mathcal{P}_{i}(\omega)\right)}\left(\omega^{\prime}\right)
\end{aligned}
$$

Hence, $E\left(u_{i}\left(a_{i}^{\prime}\right) \mid P_{i}(\omega)\right)=\frac{E\left(u_{(i, \mathcal{E})}\left(\mathfrak{a}_{(i, \mathcal{E})}^{\prime}\right)\right)}{\pi\left(P_{i}(\omega)\right)} \leq \frac{E\left(u_{(i, \mathcal{E})}\left(\mathfrak{a}_{(i, \mathcal{E})}\right)\right)}{\pi\left(P_{i}(\omega)\right)}=E\left(u_{i}\left(a_{i}\right) \mid P_{i}(\omega)\right)$. 